\title{
Medical Cybernetics for Continuous Risk Assessment and Management of Insulin Resistance and Related Complications
}

\author{
Zsolt P. Ori ${ }^{*}$ \\ Ori Diagnostic Instruments LLC, 3407 Middlebrook Dr, Durham, Nc 27705, USA, \\ ori.zsolt@oridiagnosticinstruments.com
}

\begin{abstract}
Individualized precision methods are needed for continuous noninvasive monitoring of state variables of risk indicators not just for early detection of subclinical derangements but also to monitor progress of effect of lifestyle modification and medical therapies throughout lifespan. We envision a cloud based cyber-physical system where input data come from wearable sensors along with frequently or daily use of a body-composition/ hydration-analyzer/ photoplethysmography equipped stand-up scale and these sensor data are used for state space modelling of functioning in four domains with major implications for morbidity/ mortality: 1. Cardiometabolic, 2. Cardiorespiratory, 3. Cardio vegetative, and 4. Cardiovascular functioning. The metrics in these 4 domains allow to draw trajectories into the future and to continuously assess risks of endpoints. At subclinical stage of derangements cloud computing generated feedback on a mobile app may provide for automation and self-improvement to meat daily goals of therapeutic efforts. When abnormalities reach more significant level therapies by health care providers are in order along with continued self-management of modifiable risk factors with behavior modification. This article outlines how a Medical Cybernetics centered approach can be used for continuous risk assessment/ management of insulin resistance and its connected pathological processes such as endovascular inflammation and dysfunction.
\end{abstract}

\section{CCS CONCEPTS}

- Cloud Computing; • Medical Cybernetics; • Telemedicine;

\section{KEYWORDS}

Non-invasive telemonitoring, Lifestyle management, Continuous measurement of insulin resistance, Prevention of cardiometabolic diseases

\section{ACM Reference Format:}

Zsolt P. Ori and Ori Diagnostic Instruments LLC, 3407 Middlebrook Dr, Durham, Nc 27705, USA, ori.zsolt@oridiagnosticinstruments.com. 2021. Medical Cybernetics for Continuous Risk Assessment and Management of Insulin Resistance and Related Complications. In 2021 International Symposium on Biomedical Engineering and Computational Biology (BECB 2021),

${ }^{*} \mathrm{MD}, \mathrm{MS}, \mathrm{FACP}$, Member of IEEE

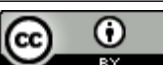

This work is licensed under a Creative Commons Attribution International 4.0 License.

BECB 2021, August 13-15, 2021, Nanchang, China

(C) 2021 Copyright held by the owner/author(s).

ACM ISBN 978-1-4503-8411-7/21/08.

https://doi.org/10.1145/3502060.3502155
August 13-15, 2021, Nanchang, China. ACM, New York, NY, USA, 5 pages. https://doi.org/10.1145/3502060.3502155

\section{INTRODUCTION}

In clinical medicine there is a need to recognize developing risks and subclinical stages of insulin resistance and associated conditions like prediabetes, diabetes mellitus (DM2) and its major complications such as cardiovascular disease (CVD) and manage recognized risks not just with medications but primarily with lifestyle modification with preventive intention in mind throughout lifetime.

For non-invasive solutions to this problem, it appears helpful to consider principles used in physics to observe seemingly random phenomena in nature in general with the intent to gain control over controllable processes. It is recognized that principles in physics are readily applicable also for exploring complex biological systems. Observer can convert prior information into prior maximum information entropy probability distribution [1]. Cybernetics add to this the element of interconnectedness of functional units and the feedback of information to each functional unit, where the outcomes of actions are taken as inputs for further action. For dynamically changing systems repeated observations/ measurements can update the a priori distribution function into posteriori one. Kalman filtering is the most appropriate algorithm that uses a series of measurements observed over time, containing statistical noise and other inaccuracies, and produces the a posteriori probability distribution over the variables for each timeframe. The state space modelling with process models and measurement models provides an appropriate framework to describe the system dynamics and allows for continuous observation of state variables [SV's] and applying optimal control strategies.

This paper discusses continuous risk assessment/ recognition of subclinical disease in the patients' home environment and wants to introduce to the reader to a paradigm shifts in risk estimation and management with the main intent of primary prevention. We want to lay out our vision how risk factors leading to endpoint diseases such as prediabetes, DM2 and its complications like CVD, could be observed through a cyber-physical system (CPS) and how selfmanagement with lifestyle modification could be supported with computer generated feedback to provide for automation and selfimprovement to meet daily goals of behavior modification efforts regarding lifestyle. The recommendation comes from [2] to shift our focus from prediction of events to detection of pre-clinical disease which could improve personalized decision-making and outcomes. Beyond risk assessment when already disease is detected the purpose remains always to offer personalized treatment to the patients with efforts for needed lifestyle improvements. The currently used methods for assessing risks such as the concept of Allostatic Load and the Framingham Risk Score are reviewed in [3]. However, these 
methods are based on in office assessments and mainly laboratory data and are performed periodically rather than continuously in the patients' home environment. They do not contain the elements of Medical Cybernetics (MC) such as continuous parametric physiological process modelling with interconnectedness and feedback. Further, they do not support individualized control of modifiable risks.

It is increasingly recognized now that the pathological processes of insulin resistance and associated conditions like of oxidative stress, endovascular inflammation and endovascular dysfunction are strongly interlinked with a commonly shared pathological pathway with potentially leading to complications such as DM2 and/ or CVD [4]. However, there is no non-invasive tool available yet to monitor insulin resistance, endovascular inflammation, and dysfunction non-invasively and continuously. With focus on this very essential issue our research and development company (ODI or Ori Diagnostic Instruments) has introduced several innovations over the years which can be employed to measure non-invasively changes of insulin resistance and associated conditions by using stochastic difference equations, cloud computing, bioelectrical and bioimpedance measurements to observe and maximize control of the state variables (SV's) of the energy metabolism [5-10]. In current paper we envision a cloud based cyber-physical system. The input data come from wearable sensors along with frequently or daily use of a body-composition/ hydration-analyzer/ photoplethysmography equipped stand-up scale (BC-HA-PG) and the sensor data are used for state space modelling in four domains of functioning with major implications for morbidity/ mortality: 1. Cardiometabolic (CMF), 2. Cardiorespiratory (CRF), 3. Cardio vegetative/ Stress (CVS), and 4. Cardiovascular (CVF). The metrics in these 4 domains allow to draw trajectories into the future and to continuously assess risks of endpoints such as DM2 and complications, and CVD. Our central proposition is that by improving insulin resistance with the use of CPS, we can ameliorate the condition of oxidative stress, overall inflammation, fat vs. carbohydrate oxidation, and CVD progression.

\section{PROPOSITION AND METHODS}

ODI's research idea is to use MC and create mathematical models of morbidity/ mortality indicators and draw trajectories of the indicators real time to observe, predict, and gain maximum control of them. The proposal of Continuous Risk Assessment and Management (CRAM) system extends ODI's innovation of a cloud based Cyber-Physical System (CPS) [6-10], a mobile technology to integrate sensory data from various mobile devices into individualized dynamic mathematical models of physiological processes allowing for analysis and prediction by machine learning for maximizing control. An overview of CRAM is in Figure 1

Figure 1. shows the flow of information. Wearable sensors of the fitness industry and BC-HA-PG pick up the needed input data. Cloud computing on virtual machines perform the model fitting to data in four domains of health: CMF, CRF, CVS, and CVF. Life-course of Trajectories can be created for risk assessment of "endpoints" and displayed on the user's Metabolic Health Monitor app (MHM). CPS can be used to calculate metrics used in Health Economics such as outcome measures like Quality Adjusted Life Year (QALY) allowing for cost effectiveness and value of information analysis (VOI). Results are displayed on the Metabolic Health Monitor app (MHM). MHM allows for planning, setting achievable goals and calculate metrics of self-efficacy in doing lifestyle changes. Feedback of information will help user to reach his/her goals. We created already the physiological process models:

Ad 1. CMF: For cardiometabolic health we use the existing fully developed Self-Adaptive Model of the Human Energy Metabolism (SAM-HEM) [5]. ODI is the first in using the principle of "least action/ stationary action" as a principle for metabolic model building of the human energy metabolism. Here we assume that the energy system uses the minimum necessary energy to arrive at next day's predicted and measured weight $\left(W_{k}, W_{k}^{\sim}\right)$, lean mass $\left(L_{k}, L_{k}^{\sim}\right)$, fat mass $\left(F_{k}, F_{k}^{\sim}\right)$ [6]. Importantly, we were able to show in an energy perturbation human experiment that our calculated Rw-ratio i.e., $R w_{k}=\Delta W_{k} / \Delta F_{k}$, were closely correlating with insulin resistance related laboratory measured HOMA-IR. During a weight loss followed by refeeding the correlation between HOMA-IR and Rw ratio was $\rho=-0.9213$ and during weight gain first followed by weight loss next the correlations was $\rho=-0.9676$ [6]. The verification of this concept was performed also using data from 12 clinical studies with 39 clinical study arms and with total number of patients $n=2010$ [7]. We were able to prove the feasibility of our modelling concept of the insulin resistance as measured with HOMA-IR by finding strong correlation $\rho=-0.6745, \mathrm{P}=0.000024$ between changes of Rw-ratio and insulin resistance HOMA-IR [7]. We performed also a sub-analysis including 3 clinical trials with a total of 11 study arms which lasted longer than 336 days. The correlation between HOMA-IR and Rw-ration was $\rho=-0.8481$ with $\mathrm{P}=0.0009699$ [7]. Encouraged by these results ODI is using now the Rw-ratio to model HOMA-IR changes. Importantly our algorithm uses only weight, fat weight, and energy balance calculations without daily macronutrient calorie intake counting [7]. Based on these results we found that our metabolic models are suitable to indirectly calculate and predict the otherwise very difficult- or impossible-to-measure slow changes of state variables (SV's) of the metabolism such as $R w_{k}$, $L_{k}, F_{k}$, fat vs. carbohydrate oxygenation $\mathrm{FatOx}_{k} / \mathrm{CarbOx_{k }}$, and non-protein respiratory quotient $24 R Q n p_{k}$ and capture them for the first time noninvasively in the user's natural environment [7].

Ad 2. CRF: We estimate maximum oxygen uptake capacity $\dot{V} \mathrm{O}_{2} \max _{k}$ which is estimated from heart rate and measuring maximal activity energy expenditure during graded exercise. The $\dot{V} \mathrm{O}_{2} \max _{k}$ calculation model is using multiple linear regression with data on age, sex, height, percent body fat, maximal activity energy expenditure, and the slope between heart rate and physical activity as in [8]. We estimate also exercise capacity $E C_{k}$, heart rate reserve $H R R_{k}$.

Ad 3. CVS: The activity of autonomous nervous system is estimated with heart rate variability: standard deviation of R-R intervals (SDNN), the ratio of the high and low frequency power spectrum components $\mathrm{HFr} / L F r_{k}$ and the average heart rate $a v H R_{k}$ in $24 \mathrm{hr}$ [8].

Ad.4. CVF: For cardiovascular functioning we want to monitor systolic $S B P_{k}$ and diastolic $D B P_{k}$ blood pressure by photoplethysmography PG. The algorithm of Shao et al [11] uses the measurement of pulse arrival time $P A T_{k}$ and the so-called MK-EE calculation 


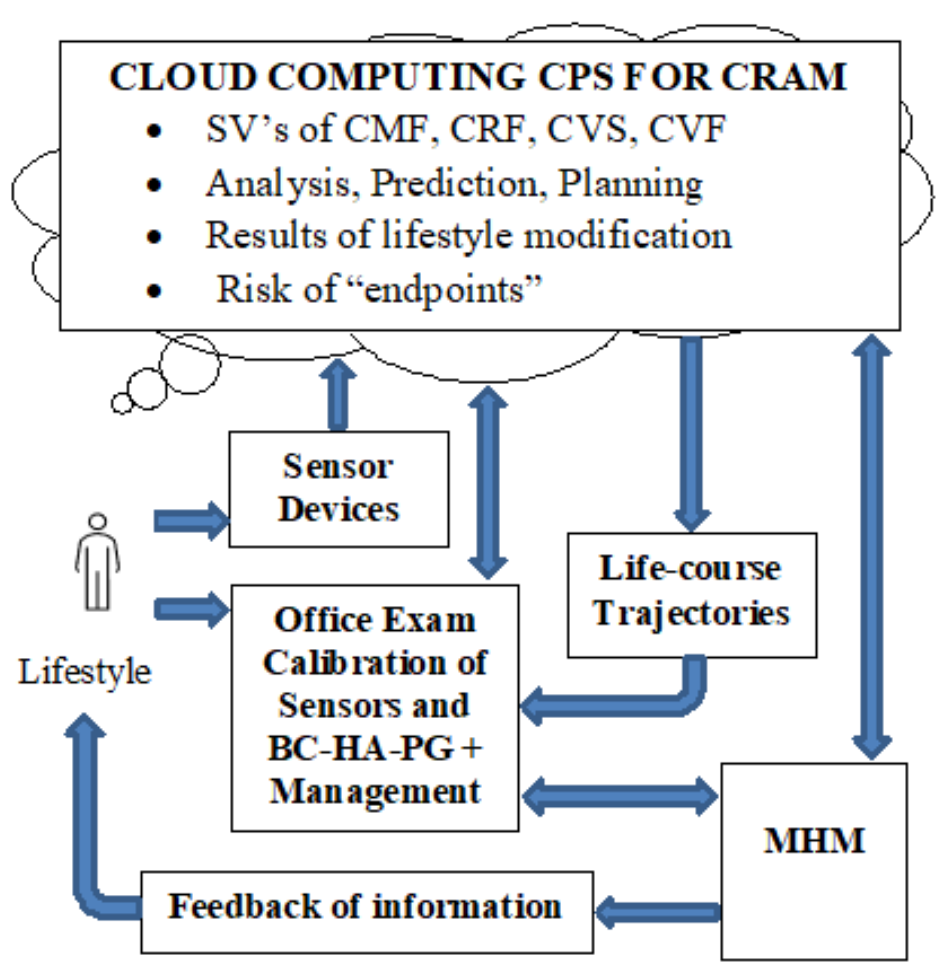

Figure 1: The Cloud Computing Cyber Physical System (CPS) for Continuous Risk Assessment and Management (CRAM). The sensor data are picked up from wearable sensors. The state variables (SV's) are constantly updated by estimators in four domains of health: CMF, CRF, CVS, and CVF. Laboratory data and office based traditional risk assessment combined with SV's can create Life-course of Trajectories allowing for assessing risk for "endpoints", providing outcome measures like QALY, health economic metrics like value of (self-) therapies allowing for value of information analysis (VOI). Results are displayed on the Metabolic Health Monitor app (MHM). MHM allows for planning, setting achievable goals and calculate metrics of self-efficacy in doing lifestyle changes.

model as in Equation (1a) and (1b) for SBP and DBP.

$$
\begin{gathered}
S B P_{k}=a_{1} \cdot \ln P A T_{k}+b_{1} \\
D B P_{k}=c_{1} \cdot \ln P A T_{k}+d_{1}
\end{gathered}
$$

The unknown parameters $a_{1}, b_{1}, c_{1}$ and $d_{1}$ are to be determined during calibration procedures. We foresee the possibility that this technology can be used in a home environment with calibration procedure during office visit.

Another indicator of CVF is the total arterial compliance $C_{T k}$ which can be estimated by measuring pulse wave velocity $P W V_{k}$ according to [12] as in Equation 2.

$$
C_{T k}=\frac{k_{1}}{P W V_{k}^{2}}
$$

In the paper of Papaioannou at al [12] the authors measure $P W V_{k}$ using tonometry to detect the pulse wave at carotid and femoral artery; the velocity calculation considers the distance between the measurement points at carotid and femoral artery as well as the transit time between proximal (carotid) and distal pressure (femoral) waveforms at their local minimums corresponding to the diastolic pressure of the wave forms. In the setting of [12] $k_{1}$ was determined to be $k_{1}=36.7$. Unfortunately, tonometry-based measurement requires highly trained technician and are not recommended for home measurements. Therefore, we consider a dual photoplethysmography measuring system for screening purpose with accepted less accuracy than tonometry for estimating the pulse arrival time at fingertip $f P A T_{k}$ and toe tip $t P A T_{k}$ and calculate $P W V_{k}$ from the velocity by dividing the distance between fingertip and toe tip $D F T_{1}$ by the time difference of arrival of pules waves $t P A T_{k}-f P A T_{k}$ as in (3).

$$
P W V_{k}=\frac{D F T_{1}}{t P A T_{k}-f P A T_{k}}
$$

Individualized calibration might help to improve the accuracy compared with the currently accepted gold standard tonometry and find the applicable parameter $k_{1}$ with tonometer during office visit.

\section{HOME MEASUREMENTS}

For home use we envision a Body-Composition Hydration-Analyzer Photoplethysmography equipped stand-up scale (BC-HA-PG) to supply daily measurement of weight $W_{k}^{\sim}$, lean mass $L_{k}^{\sim}$, fat mass $F_{k}^{\sim}$, 


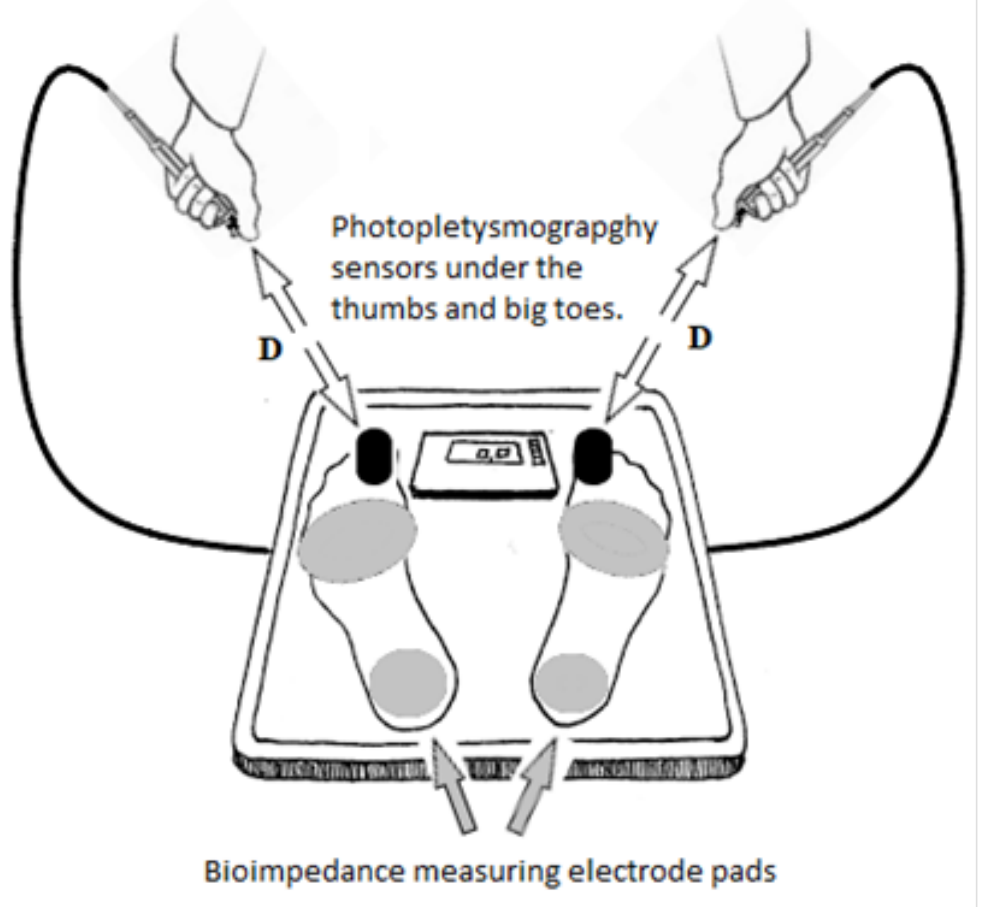

Figure 2: The Body-Composition Hydration-Analyzer Photo-plethysmography equipped stand-up scale.

extracellular water mass $E C W_{k}^{\sim}$, intracellular water mass $I C W_{k}^{\sim}$, and the pulse arrival time at toe tip $t P A T_{k}^{\sim}$ and fingertip $f P A T_{k}^{\sim}$ as in 'Figure 2'. Note in the design of BC-HA-PG the handheld grips at the tip have a photoplethysmography sensor installed under the thumbs. Likewise, the big toes are lying over photoplethysmography sensors as well. The sketch also shows the electrode pads of the bio-impedance stand-up scale for measurement of body composition and hydration status. Arms are in a relaxed potion next to the body with well-known measured distance $\mathbf{D}=D F T_{1}$ between thumb and big toe.

We already have patented solutions to realize the hardware and software of the BC-HA part of the stand-up scale [9]. The main function of the PG part to measure the pulse arrival time at fingertip $f P A T_{k}^{\sim}$ and toe tip $t P A T_{k}^{\sim}$. The former can be measured with a PG sensor device placed to the end of a handheld grip which is wire connected to BC-HA-PG and the latter by a PG sensor touching the big toe which could be an integral part of the stand-up scale. Knowing $t P A T_{k}^{\sim}$ and $f P A T_{k}^{\sim}$ allows for calculating $P W V_{k}$ from the signals using equation 4 ) and knowing the distance $\mathrm{DFT}_{1}$ between the two sensors of PG. The measurement results $t P A T_{k}^{\sim}$ and $f P A T_{k}^{\sim}$ are used both to calculate $S B P_{k}, D B P_{k}$, and $C_{T k}$ as in equations (1a), (1b), (2), and (3), respectively. In office measurement with tonometry could help calibrate the measuring algorithm.

\section{PREDICTIVE EQUATIONS OF CONTINUOUS RISK ASSESSMENT AND MANAGEMENT}

Our Risk Assessment and Management (CRAM) system is using state-space modelling with stochastic difference equation similarly to calculation of a trajectory of a satellite [13]. For process modelling and creating the Life-course Trajectories in the four physiological domains (CMF, CRF, CVS, CVF) equation (4a) and for measurement modelling equation (4b) are used as on page 269 in [13].

$$
\begin{gathered}
x_{k+1}=\Phi(k+1, k) x_{k}+\Gamma(k) w_{k+1} \\
y_{k}=M(k) x_{k}+v_{k}
\end{gathered}
$$

Here $x_{k}$ is a state variable vector (SV). $y_{k}$ is the measurement vector; $\Gamma(k)$ is the system noise coefficient matrix; $w_{k+1}$ and $v_{k}$ are white Gaussian sequences.

For CMF we have published already the process equations for daily changes of the body composition [5]. The SV vector is $C M F x_{k}=\left\{R w_{k}, L_{k}, F_{k}, F a t O x_{k} / C a r b O x_{k}, 24 R Q n p_{k}\right\}$; the SV for CRF is $C R F x_{k}=\left\{\dot{V} O_{2} \max _{k}, E C_{k}, H R R_{k}\right\}$, (see also [8]); the SV for CVS is $C V S x_{k}=\left\{S D N N_{k}, \frac{H F r}{L F r}{ }_{k}, a v H R_{k}\right\}$ (see also [8]); and the SV for CVF is $C V F x_{k}=\left\{S B P_{k}, D B P_{k}, C_{T k}\right\}$. The use of the Kalman filter helps to update the process models during regular use of home measurements. The measurement models could be calibrated to acceptable office-based measurement methods as indicated in 'Figure 1' during Office Exam. For example, the home measurement of variables of $C M F x_{k} R w_{k}$ can be calibrated to HOMA-IR, $L_{k}, F_{k}$ can be calibrated to DEXA scan measurements, $\mathrm{FatOx}_{k} / \mathrm{CarbOx}_{k}, 24 R Q n p_{k}$ can be calibrated to metabolic chamber measurements. $C R F x_{k}$ could be calibrated to cardiopulmonary exercise measurement of $\dot{V} \mathrm{O}_{2} \max _{\dot{k}}^{\sim}$ which is the "gold standard" measurement of $\dot{V} \mathrm{O}_{2} \max _{k}^{\sim}$. For measuring CVS there is no "gold standard". Therefore, we use published age adequate values for CVS to compare the home measured $C V S x_{k}$ with. Regarding CVF the "gold standard" is to measure coronary artery blood flow reserve. An 
acceptable office-based procedure is the flow mediated dilatation measurement and the measurement of total arterial compliance [12] using tonometry.

The risks of insulin resistance related conditions such as cardiometabolic diseases and CVD are competing causing morbidity and mortality calling for analysis for Cumulative Incidence in the presence of Competing Risks (CICR). The mathematical tools are summarized in [14]. The overall goal is to arrive at individualized probability density functions of an endpoint such as complication or death. An example for how to do this is given in [15].

Before a long-time observational database can be acquired from home measured SV's the individual risks could be estimated with already available software tools. The SV's could be directly plugged into available software products which have been developed with the best available evidence and have been validated against clinical trial data such as in [16] to estimate risks of endpoints. These include among others all-cause mortality, incident rate for each complication, micro- and macrovascular CVD risk, any type of stroke, any type of coronary event, any type of heart failure. It is foreseeable that data collected by CPS and the CRAM system would allow for measuring value in primary prevention and primary care including outcome measures such as QALY, value measurements with incremental cost-effectiveness ratio (ICER).

\section{DISCUSSION}

It is recognized in this paper that the available bioengineering literature has not embraced yet the concept of continuous risk assessment and management of insulin resistance and related complications for the public and clinical use. This important concept could widely extend the horizon of Medical Cybernetics with implications for individualized health care and how primary care/ public health is provided.

We provided here a broad overview how Medical Cybernetics can be used not just for continuous risk assessment but also as a tool which can create a Life-course Trajectory of a disease condition and give metrics in several functional domains which are intricately connected to major morbidity and mortality.

The metrics will allow to track progress of lifestyle modification which is needed for prevention and support treatment of cardiometabolic derangements. SV's along with its Life-course Trajectory displayed on the user's Metabolic Health Monitor mobile app will give opportunity for learning about risks for health and developing new skills to fight risks, increase motivation for lifestyle change, allow for measuring self-efficacy in the fight against modifiable risks, allow for receiving planning guidance from trainers/fitness coaches and healthcare provider(s). The self-explaining context of SV's have the potential to raise self-awareness and draw attention to risk reduction and individual responsibility in the fight against insulin resistance related disease processes. With user consent, the large volume of impactful data could be donated for research purposes to fight insulin resistance and related complications.

The future prospective of our Cyber Physical System for Continuous Risk Assessment and Management is that it opens a new field of research using tools of Medical Cybernetics. Principles of continuous non-invasive risk assessment and management of health in the user's home environment could be followed realizing individualized "precision medicine". We envision the possibility that health care related value metrics can be derived opening the possibility of big data research with high power computing/ machine learning delivering needed information to life science research and payors while patients' interests and values are kept in the focus. The feedback of individualized metrics along with using tools of the digital health technologies may amount to channelling focus also to effective and cost-effective individualized care and to accelerating lifestyle and nutrition science. CPS may facilitate achieving important priorities of community/ public health goals which cannot be readily accomplished through traditional approaches.

\section{ACKNOWLEDGMENTS}

This research was supported by Ori Diagnostic Instruments, LLC. We would like to thank Ilona Ori, JD, Ori Diagnostic Instruments, LLC, for editorial help.

\section{REFERENCES}

[1] Crnkovic G D. 2012 Information and Energy/Matter. Information 3(4) 751. doi.org/10.3390/info3040751

[2] McEvoy J W et al. 2014 Risk and the physics of clinical prediction Am 7 Cardiol. 113(8) 1429. doi:10.1016/j.amjcard.2014.01.418

[3] Levine ME, Crimmins EM. 2014 A comparison of methods for assessing mortality risk. Am f Hum Biol. 26(6) 768. doi: 10.1002/ajhb.22595

[4] Ceriello A et al. 2004 Is Oxidative Stress the Pathogenic Mechanism Underlying Insulin Resistance, Diabetes, and Cardiovascular Disease? The Common Soil Hypothesis Revisited Arterioscler Thromb Vasc Biol. 24 816. doi: 10.1161/01.ATV.0000122852.22604.78

[5] Őri Z. 2016 Parametric recursive system identification and self-adaptive modeling of the human energy metabolism for adaptive control of fat weight. Medical and Biological Engineering and Computing 55(5) 759 doi: 10.1007/s11517-016-1552-3

[6] Ori, Z. 2019 Cyber-Physical System for Management and Self-Management of Cardio-metabolic Health. IntechOpen on-line http://dx.doi.org/10.5772/ intechopen. 84262

[7] Ori, Z 2019 Metabolic Health Analysis and Forecasting with Mobile Computing. IntechOpen on-line doi: 10.5772/intechopen.88872

[8] Ori, Z 2020 Integrated Cyber-Physical System to Support Early Diagnosis and Prevention of Prediabetes and Complications of Type 2 Diabetes IntechOpen on-line doi: 10.5772/intechopen.94232

[9] Óri Zs 2018 U.S. Patent No.: 9,949,663 B1 Date: 4/24/2018, No.: 10,716,491 B2 Date: 7/21/2020, No.: 10,653,333 B1 Date: 5/19/2020; No.: 10,987,019 B2 Date: 4/27/2021

[10] Ori, Z, Ori I: 2021 Principles of Continuous Risk Monitoring of Body Composition, Insulin Resistance, Endothelial Dysfunction and Nutrition to Improve General Health and Prevent Cardiovascular Disease and Cancer IFISET Vol. 8 Issue 4, April 2021 ISSN (Online) 2348 - 7968, www.ijiset.com

[11] Shao A J, et al. 2020 An Optimization Study of Estimating Blood Pressure Models Based on Pulse Arrival Time for Continuous Monitoring f Health Eng. 101078251. doi: $10.1155 / 2020 / 1078251$

[12] Papaioannou G T, et al 2017 Total arterial compliance, estimated by a novel method, is better related to left ventricular mass compared to aortic pulse wave velocity: The SAFAR study Clinical and Experimental Hypertension 39(3), 271 doi:10.1080/10641963.2016.1247165

[13] Jazwinski H A 2007 Stochastic processes and filtering theory. (Dover publications, Inc. Mineola, New York) p 269

[14] Kim H T 2007 Cumulative incidence in competing risks data and competing risks regression analysis. Clin Cancer Res. 13(2 Pt 1) 559 doi:10.1158/1078-0432.CCR06-1210

[15] Sakamoto M, et al. 2018 The impact of creating mathematical formula to predict cardiovascular events in patients with heart failure Sci Rep. 8(1) 3986 doi:10.1038/s41598-018-22347-0

[16] Palmer A J, 2013 Computer modeling of diabetes and its complications: a report on the Fifth Mount Hood challenge meeting. Value Health. 16(4) 670 doi: 10.1016/j.jval.2013.01.002 